\title{
O ENCONTRO (PÓS-COLONIAL): "RAÇA”, HISTÓRIA E TERRITÓRIO NO BRASIL, ÁFRICA DO SUL E CARIBE
}

Fernando Rosa Ribeiro ${ }^{1}$

Trabalhando comparativamente há anos com construções de "raça" e nação, ficou claro para mim que esses construtos não só divergem no tempo, como também no espaço (Rosa-Ribeiro 1996a, 1998, 2000, 2002a, 2002b). Além do mais, encontram-se invariavelmente inseridos dentro de percepções da história que não são convergentes. Pelo contrário, frequientemente são dificilmente comparáveis entre si. Contudo, como áreas de antiga colonização européia, tanto Brasil como África do Sul e a região do Caribe possuem discursos que, ainda que diversos, são comparáveis em alguns pontos. Isto apesar de existirem, naturalmente, inúmeras dificuldades na comparação de tradições discursivas e práticas sociais inseridas em contextos (pós-) coloniais diversos, línguas diferentes e tradições acadêmicas distintas.

"Raça" e práticas racistas, por exemplo, existiram e existem em todas as três regiões. Em minha tese de doutorado (Ribeiro 1996a) tentei comparar as construções de "raça" e a discriminação racial na África do Sul e Brasil. Ficou claro que, apesar de ambos apresentarem construtos relacionados a "raça" e práticas sociais racistas, estes divergiam enormemente de um país para outro. Ambos os países construíram ademais narrativas poderosas a respeito de si próprios, enraizadas em historiografias nacionais. Por exemplo, um elemento fundamental da auto-imagem brasileira é de que o país, apesar de não ser uma "democracia racial" freyreana, possui contudo práticas sociais

${ }^{1}$ Professor Doutor da Universidade Federal de Goiás

Universitas - Relações Int., Brasília, v. 2, n.2, p. 83-107, jul./dez. 2004 
relacionadas a cor que divergem fundamentalmente das americanas ou sul-africanas (Banton 1967, Degler 1971, Skidmore 1974, 1993, Souza 1997). O artigo de DaMatta (1981) sobre a "fábula das três raças" é essencial aqui - isto é, a narrativa segundo a qual a nação brasileira teria se construído através do esforço conjunto, miscigenação e mestiçagem cultural entre o branco (português), o negro (africano) e o índio. Nas narrativas nacionais brasileiras, o contraste com os Estados Unidos e a África do Sul - construídos como "outros" racistas por excelência - é muito importante, como por exemplo na obra freyreana (Freyre 1958, Araújo 1994, Ribeiro 2000). Interessantemente, também no discurso de apartheid em língua afrikaans há uma reivindicação de diferença com relação ao discurso anglo-saxão (construído como "liberalismo" ou "imperialismo" britânicos - Coertze 1943, Cronjé 1945, 1946, 1948, Rhoodie 1969, Coetzee 1991, Rosa-Ribeiro 1996a, 1998). Isto é, embora constituídas de maneira muito diferentes, tanto as narrativas afrikaans quanto as brasileiras "conversam" entre si, embora não diretamente, mas sim através de um outro anglo-saxão. Outra semelhança entre esses dois conjuntos tão díspares de narrativas é que ambos se fundamentam em pensamentos de origem ocidental ou européia. Uma diferença importante entre os pensamentos de origem ocidental em ambos os países é contudo o fato de que, embora a África do Sul figure em narrativas brasileiras (como um outro racista), o Brasil não figura em narrativas sul-africanas. As narrativas brasileiras referem-se a "raça" e racismo freqüentemente através da figura de um outro construído - os Estados Unidos e a África do Sul (ambos possuem aqui papéis intercambiáveis). Contudo, o discurso sulafricano, apesar de mencionar um outro anglo-saxão, é um discurso que se refere primordialmente à própria África do Sul - ademais, é claramente um discurso racial ligado a uma política racial (apartheid) levada a cabo pelo Estado. O mesmo não se dá com as narrativas brasileiras: apesar de estas mencionarem as "raças" (como na fábula descrita por DaMatta), elas o fazem em termos míticos. Isto é, elas não constituem um discurso articulado sobre "raça" no modelo sul-africano ou norte-americano, e muito menos se relacionam a uma política "racial" levada a cabo pelo Estado.

Universitas - Relações Int., Brasília, v. 2, n.2, p. 83-107, jul./dez. 2004 
Uma outra diferença importante do Brasil com relação à África do Sul tem a ver com "raça" também: manter-se com uma identidade racial branca, no modelo do discurso racial europeu e sul-africano, foi muito difícil no Brasil. Uma identidade branca dificilmente poderia ser literal, como na África do Sul. Historiadores mostram que o Brasil colonial já era em grande medida uma sociedade mestiça (Alencastro 2000, Degler 1971). Embora os pensadores e intelectuais brasileiros tivessem conhecimento íntimo das teorias raciais euro-americanas, sabiam também que a realidade brasileira era diversa (Ortiz 1986, Schwarcz 1993, Skimore 1993, 1994). Assim, uma identidade branca nunca pôde ser um construto tão poderoso como o foi na África do Sul - se não fosse por outra razão que o fato de que a população dita mestiça era muito grande. Mesmo políticos como José Bonifácio já se davam conta nos primórdios do século XIX da importância da população livre de cor na construção de uma nação brasileira (Silva 1999). Não é que ser branco não fosse um valor importante. Pelo contrário, toda a evidência histórica aponta para o fato de que sim era (e é) (Azevedo 1987). Contudo, mesmo com a imigração européia maciça do século XIX e inícios do XX, uma nação branca no modelo europeu simplesmente não era possível (Torres 1982 [1914]). Embora o sonho do "embranquecimento" nunca fosse realmente abandonado (como não o foi por exemplo nem por Gilberto Freyre - Ribeiro 1996a), ele foi "sublimado", por assim dizer, na figura ou tropo do mestiço: através da miscigenação e da mestiçagem (cultural e "racial"), surgiria a nação brasileira (Skidmore 1974, DaMatta 1981, Munanga 1999, Ribeiro 1993, 1996a). Essa idéia - estranha de um ponto de vista sul-africano - foi poderosa no Brasil. Como DaMatta sugeriu em seu ensaio famoso, em comparação com os Estados Unidos (ou África do Sul), o contato foi supostamente a norma no Brasil, em lugar da segregação. Isto é, nessa interpretação o racismo, apesar de comum e geral, nunca ganhou expressão jurídica na lei e numa separação altamente formalizada como nos Estados Unidos ou na África do Sul. Assim, a discriminação, embora predominasse, não dependeu de um discurso racial ligado a uma política racial levada a cabo pelo Estado (Woodward 1957, Beinart and Dubow 1995, Worden 1994, Hasenbalg 1979, Agier 1994, Datafolha 1995, Ribeiro 1996a, Souza 1997). Pelo

Universitas - Relações Int., Brasília, v. 2, n.2, p. 83-107, jul./dez. 2004 
contrário, em lugar de ser de estatuto, a discriminação está enraizada na prática social (enquanto que na África do Sul ela esteve historicamente ancorada tanto no estatuto quanto na prática social).

Apesar da existência de outras narrativas e tropos (especialmente a nível local), o tropo historicamente dominante foi o da mestiçagem ou miscigenação, o que impediu o aparecimento de identidades raciais muito bem demarcadas e definidas como na África do Sul. Naturalmente, existem, por exemplo, identidades negras no Brasil (Sheriff 1997, Figueiredo 1998, Marc 1998). Estas contudo estão construídas sem referência a um arcabouço jurídico discriminatório ou a uma política de Estado racializada, pelo menos a partir da abolição. Ao contrário das narrativas sul-africanas, onde a figura do mestiço é explicitamente rejeitada (Cronjé 1945, Coetzee 1991, Ribeiro 1996a), nas narrativas brasileiras a diferença original do "branco", "negro" e "índio" é transcendida através de uma identidade comum e homogeneizante, a do mestiço (Ribeiro 1995, DaMatta 1997, Munanga 1999). Este fato é muito importante - essencial mesmo numa comparação com a situação sul-africana. Nesta, houve o que se chamou de white supremacy, a "supremacia branca", um construto originário do sul dos Estados Unidos importado para descrever a situação sul-africana. Embora historicamente dominantes no Brasil, os brancos não constituíram uma "supremacia branca" através de uma política racial (Banton 1967, Frederickson 1981). Em termos de uma mimese colonial, portanto, os brancos brasileiros e sul-africanos apresentam padrões altamente divergentes de construção de identidade (Taussig 1993, Bhabha 1994, Ribeiro 1996a, 2000).

Embora existissem mestiços na África do Sul colonial, eles eram um grupo social demograficamente muito mais restrito e localizado do que no Brasil (Elphick e Giliomee 1988). Isto se deve a vários fatores históricos. Em primeiro lugar, o colonialismo na África do Sul - holandês e, após 1815, inglês - é de origem mais recente do que no Brasil (a União da África do Sul só surgiria em 1910, e a emancipação da tutela legal, parlamentar e diplomática britânica só a partir de 1934). Ademais, a África do Sul, a contrário do Brasil, a partir de 1815 foi colônia de um império prestigioso e em franca expansão (Davenport 1987, Worden 1994). Enquanto que o Rio de

Universitas - Relações Int., Brasília, v. 2, n.2, p. 83-107, jul./dez. 2004 
século XIX, por exemplo, foi uma capital com maioria de população escrava ou livre de cor, com uma pequena minoria branca (Debret e Alencastro 2002), a Colônia do Cabo da Boa Esperança (embrião histórico da atual África do Sul) era até meados do século XIX uma colônia onde colonos de origem européia chegaram a ser maioria, e sempre foram uma população numericamente muito importante. Isto só se modificou substancialmente ao longo do século XIX, com a expansão territorial para além dos rios Orange e Vaal. Esse processo ficou conhecido como Grande Trek, ou grande migração para o interior (Muller 1982). Embora a colônia do Cabo tivesse escravidão, era muito menos importante do que no Brasil, que foi a maior colônia escravocrata da história moderna. Ademais, embora existissem fazendas, o sistema de plantation nunca se configurou no Cabo (Worden 1985).

Recriar ou reconstruir identidades brancas européias no Cabo foi portanto um projeto muito mais viável do que no Brasil (Ross 1994, Hofmeyer 1987, O'Meara 1983, Du Toit and Giliomee 1983, Giliomee 1989, Van Jaarsveld 1959, 1984, Huigen 1996). Ademais, o status colonial até bem entrado o século XX assegurou uma importante influência metropolitana em assuntos internos, o que permitiu que a manutenção de uma identidade européia fosse mais viável ou mesmo necessária dentro do sistema de relações imperiais britânicas (no qual a África do Sul, no século XX, passou a integrar o grupo seleto dos chamados white dominions, junto com o Canadá, a Austrália e a Nova Zelândia, todos países de clara maioria branca, ao contrário da África do Sul). Se aceitarmos a interpretação de Elphick e Giliomee (1988) da história da colônia do Cabo, apesar da vasta expansão territorial efetuada durante o século XIX e as importantes descobertas de minérios a partir de 1870 (ouro e diamantes), e a conquista de várias sociedades africanas durante todo o século XIX (Davenport 1987, Worden 1994), o padrão estabelecido no Cabo continuou após 1840 colonização branca, pouca ou nenhuma miscigenação, pequena população livre de cor (antes de 1840), e, principalmente pouca ou nenhuma incorporação de não-europeus no grupo europeu (Elphick e Giliomee 1988, Macmillan 1968, Biewenga 1999, Ribeiro 1998).

Universitas - Relações Int., Brasília, v. 2, n.2, p. 83-107, jul./dez. 2004 
Além do mais, a presença de populações autóctones muito numerosas também teria impedido qualquer política de extermínio ou, inversamente, de assimilação, como a levada a cabo no Brasil com os indígenas (Puntoni 1988). Ou melhor, embora tanto extermínio como assimilação houvessem existido, a longo prazo e no país como um todo a eficácia dessas estratégias foi comparativamente muito mais limitada no caso sul-africano (Van der Merwe 1937). No Brasil, as populações indígenas e autóctones provavelmente não constituem hoje sequer $1 \%$ da população total, enquanto que os africanos autóctones perfazem bem mais de dois terços da população total da África do Sul. Portanto, em comparação com o caso brasileiro, na situação sul-africana um discurso racial ligado a uma política racial tiveram um papel importantíssimo. Este discurso desenvolveu-se contra o pano de fundo da percepção colonial de uma ampla "massa" de "nativos" que não se considerava que fizessem parte da nação colonial e, mais tarde, póscolonial (Coertze 1943, Marks e Trapido 1987, Dubow 1984, 1987, 1989, 1993); e desenvolveu-se ademais dentro do bojo de um enorme império transnacional em expansão, cuja metrópole e white dominions agiam como uma espécie de "fiadores" da identidade branca local. Isto é, essa identidade tinha um importante respaldo internacional e diaspórico. $\mathrm{Na}$ imaginação colonial e pós-colonial sul-africana, o mestiço - denominado Coloured - em lugar de ser a base para a construção da nacionalidade e da nação, como no caso brasileiro, ou, seguindo a interpretação de DaMatta (1981), o ponto no qual as diferenças se encontravam criando a nação comum, tornou-se uma espécie de minoria étnica com um estatuto extremamente ambíguo dentro da construção de uma nação sul-africana (Patterson 1953, February 1981, Goldin 1987, James 1996, Rosa-Ribeiro 1993, 1996a, 1996b).

Contudo, apesar de diferenças, tanto o discurso racial sulafricano quanto as narrativas brasileiras têm raízes profundas na episteme ocidental (Foucault 1966, 1969). O fato de que os dois lados não conseguem facilmente se reconhecer um no outro ocultou isso, assim como o fato de que historicamente ambos os países pertenceram a esferas imperiais distintas e tiveram pouco ou nenhum contato entre si. Contudo, a preocupação com a construção de uma nação

Universitas - Relações Int., Brasília, v. 2, n.2, p. 83-107, jul./dez. 2004 
homogênea é uma característica comum aos dois, apesar das diferenças importantes nas respectivas estratégias de construção nacional. As elites de ambos os países consideraram historicamente a presença de vastas populações não-européias no espaço nacional como um problema que exigia uma solução. Ambos os países assumiram, modificaram e adaptaram construções européias de diferença cultural e "racial", mesmo que os resultados pareçam muito diferentes em cada caso. Finalmente, ambos os países subordinaram os não-europeus dentro do espaço nacional, mesmo que essa subordinação se tenha dado historicamente por meio de estratégias e construtos muito dessemelhantes entre si (Ortiz 1986, Marks e Trapido 1987, Beinart e Dubow 1995, Ribeiro 1996a). Contudo, essas semelhanças de fundo entre os dois países geralmente não vêm à tona devido ao grande poder persuasivo dos respectivos discursos, que marcam muito a diferença nacional e a especificidade local.

Incluir a região do Caribe na comparação é um desafio maior ainda do que comparar o Brasil com a África do Sul. O Caribe apresenta vários problemas espinhosos para o pesquisador (mesmo para aquele que não está interessado numa perspectiva comparativa). Em primeiro lugar, está a imensa variedade da região e seu caráter altamente compartimentado, que surge em toda a literatura especializada: há um Caribe anglófono, um Caribe francófono, um Caribe de colonização holandesa, e um Caribe hispânico (neste último vive mais de dois terços da população da região) (Hoetink 1967, Reno 1987, Knight 1990, Ribeiro 2002a). Ao mesmo tempo, apesar de sua imensa fragmentação, argumenta-se que a região possua um discurso comum (Glissant 1981). Embora tanto o Brasil como a África do Sul tenham experimentado a colonização por mais de um poder colonial, nenhum dos dois países possui a experiência de ter sido colonizado por vários poderes ao mesmo tempo ao longo de toda a sua história, incluindo a época dita pós-colonial (Mello 1997, Davenport 1987, Elphick e Giliomee 1988). Talvez somente no Sudeste Asiático insular e no Pacífico é que podemos encontrar outra região semelhante (Ribeiro 2001a). Os diferentes tipos de colonização surgidos no Caribe não só criaram diferentes bolsões, por assim dizer, dentro da região, como tornaram a unidade regional mais difícil de se discernir. Além do

Universitas - Relações Int., Brasília, v. 2, n.2, p. 83-107, jul./dez. 2004 
mais, o colonialismo na região teve um caráter peculiar em comparação com o colonialismo que predominou no continente americano.

Ao contrário do continente, a presença de um determinado poder colonial num território ou ilha caribenhos não determina a transmissão da cultura, língua ou religião metropolitanas para aquele território. Assim, as ilhas holandesas de sotavento (Aruba, Bonaire e Curaçau) são católicas e falam papiamento (uma língua crioula de base léxica hispânica); as antigas Antilhas Dinamarquesas tinham um crioulo de base léxica holandesa como língua comum (e atualmente, sob o nome de Virgens Americanas, têm o inglês como única língua); Trinidad era uma ilha espanhola com população de língua francesa e créole (crioulo de base léxica francesa), que emigrara para ali depois da revolução haitiana. Com a conquista inglesa em 1797, passou ao domínio britânico, mas continuou se mantendo ao longo do século XIX como ilha de fala francesa e créole, situação que perdurou até o limiar do século XX. Neste século, tornou-se uma ilha de língua inglesa e maioria de população de origem indiana. Saint Martin, nas Pequenas Antilhas, é uma ilha dividida entre uma parte francesa e outra holandesa, mas a língua principal da ilha é o inglês (sendo que nunca foi colônia inglesa), e não é católica (como a França) nem calvinista (religião historicamente dominante na Holanda), mas sim metodista e anglicana. O Suriname, colônia holandesa, ao longo de sua história freqüentemente teve uma maioria de colonos europeus de origem judia, e mesmo entre os brancos o holandês não era língua muito falada antes do século XX. A população calvinista era uma minoria, sendo que os judeus e moravianos (entre os escravos e libertos) predominavam. Além do mais, o pacto colonial funcionava mal ou simplesmente não se aplicava: a Martinica, por exemplo, uma ilha francesa, costumeiramente comerciava com os holandeses, quebrando assim o monopólio comercial francês. Os holandeses eram, ademais, os comerciantes e fornecedores de preferência em todo o Caribe, assim como os judeus eram os grandes financiadores de empréstimos de capital na região, fosse qual fosse a colônia. Latifundiários com capital, equipamentos e escravos freqüentemente se deslocavam de uma colônia a outra, em busca de melhores oportunidades, não

Universitas - Relações Int., Brasília, v. 2, n.2, p. 83-107, jul./dez. 2004 
importando se a nova colônia fosse de outro poder colonial ou formalmente de outra religião. Finalmente, principalmente nos séculos XVII e XVIII, a autonomia colonial era enorme: em Barbados, por exemplo, houve casos de governadores enviados pela metrópole serem mandados embora logo ao desembarcarem, a metrópole sendo obrigada a aceitar um governador nomeado localmente; no Suriname, conflitos entre governadores nomeados na metrópole e os latifundiários mais ricos eram comuns a ponto de efetivamente relativizarem o controle metropolitano (Schalkwijk 1994). Trata-se portanto de uma região historicamente profundamente fragmentada e plural, e marcada ademais por grandes fluxos migratórios, que transformariam no século XX algumas ex-colônias, como Trinidad, Guiana e Suriname, em países de maioria de população descendente de asiáticos (indianos, chineses e javaneses) e outras em grande centro de atração de imigrantes (como Aruba ou Saint Martin), a ponto de a população local ficar em minoria relativamente à descendente de imigrantes.

Das três regiões, o Caribe é portanto a que coloca os problemas mais espinhosos para o investigador. Após a abolição da escravatura na região (ocorrida entre 1834 e 1886), exceto no Caribe hispânico, com a decadência da economia de plantation, as elites brancas ou deixaram gradualmente a região (como no caso do Suriname), ou tornaram-se minorias demograficamente quase insignificantes. Eventualmente, a partir da década de 1930, após uma longa luta que durou grande parte do século XIX e início do XX, os negros e asiáticos passariam a ter o poder polítco (Knight 1990, Schalkwijk 1994). Além do mais, a difusão da ensino universal, logo após as diversas abolições, seguindo modelos metropolitanos, aliada ao tamanho minúsculo da elite branca local, permitiu o surgimento de uma classe-média mulata e, em seguida, negra, em todo o Caribe, de onde saíram os líderes caribenhos de meados do século XX. Esse movimento emancipatório não significou, contudo, uma liberação das epistemes de origem ocidental (Henry 2000, Wilson-Tagoe 1998, Sankatsing 1989). Não obstante, significou que os pensadores e intelectuais negros e asiáticos da região geralmente gozaram - e gozam - de um status muito maior do que foi o caso no Brasil ou na África do Sul. Além do mais, esses intelectuais

Universitas - Relações Int., Brasília, v. 2, n.2, p. 83-107, jul./dez. 2004 
puderam desenvolver uma crítica muito mais contundente do pensamento europeu (James 1963, Fanon 1967, Harris 1967, De Kom 1981 [1934], Glissant 1989, Sankatsing 1989, Wilson-Tagoe 1998, Henry 2000). Finalmente, antes da Segunda Guerra Mundial, houve uma efervescência política e intelectual no Caribe, acompanhada de movimentos sociais, que afetou inclusive os Estados Unidos e sua população negra (James 1998).

Outra característica importante do Caribe é que este não é um estado-nação no sentido brasileiro ou sul-africano. Tampouco é propriamente uma coleção de estados-nação. A meu ver, é melhor considerar o Caribe como um espaço aberto de incorporação onde categorias como "nação", "estado", "raça", "colonialismo", embora longe de estarem ausentes, têm um peso diferenciado com relação a outras regiões (Van Lier 1971, Ribeiro 2002a). Esse espaço pode ser pensado como uma fronteira. Esta é um espaço tanto imaginado quanto concreto. É marcado por fluxos diaspóricos intensos - migrações internas e externas - vinculados a tradições rizomáticas (Chamberlain 1998, Goldin 1999). Isto é, aqui a tradição não está fixada, mas sim moldada a partir de várias fontes ao mesmo tempo: ela se constrói e surge "lateralmente", por assim dizer, como um rizoma, para empregar a imagem de Deleuze tal como apropriada por Braidotti (1995). A fronteira portanto aparece rizomaticamente: ela é o locus onde as identidades surgem. Seguindo Homi Bhabha, a fronteira é onde algo novo começa a surgir, o "entrelugar" (Bhabha 1994). Bhabha propõe, fundamentando-se em Heidegger, que a fronteira é onde algo começa a fazer-se presente. É o locus do encontro por excelência, um espaço residual onde se desenvolve um excedente, isto é, algo que não estava lá antes do encontro (e que não é a mera soma dos elementos precedentes). Nesse sentido, poderíamos dizer que a diáspora é o surgimento no espaço de uma cultura rizomática: é um encontro entre diferentes tipos de alteridade, encontro a partir do qual algo novo surge.

O Brasil ou a África do Sul têm fronteiras internas. Estas foram fronteiras definidoras da nação e da nacionalidade, além de loci de violência: basta pensar no sertão brasileiro e o poderoso imaginário a

Universitas - Relações Int., Brasília, v. 2, n.2, p. 83-107, jul./dez. 2004 
ele vinculado (Velho 1976, Moog 1978, Rosa 1980 [1956], Cunha 1985 [1902], Puntoni 1998, Souza 1998, Suárez 1998); ou ainda no interior sul-africano e o imaginário a ele relacionado (Walker 1930, Van der Merwe 1937, 1995, MacCrone 1937, Legassick 1980, Elphick and Giliomee 1988, Coetzee 1988, Huigen 1996, Rosa-Ribeiro 1998). Ao ler o ensaio de René Achéen sobre a história das Antilhas Francesas (Achéen 1983), compreende-se que as ilhas sofreram o que Achéen denomina de "colonização total". Isto é, ao contrário do que aconteceu no Brasil e na África do Sul, onde sempre houve uma fronteira a ser ocupada. que poderia servir de local de refúgio e, o que é mais importante, como horizonte imaginativo da nação, nas Antilhas (especialmente nas Pequenas Antilhas), não havia possibilidade de fuga ou recuo no espaço, a não ser para as chamadas mornes ou encostas de terra de má qualidade, onde por vezes escravos e livres se refugiavam. Isto é, devido à exigüidade do território, a não ser que se lançasse ao mar ou passasse a uma ilha vizinha, não havia possibilidade concreta de fuga. O espaço da fronteira que é "vazio" (pelo menos na imaginação), e que foi tão importante na história brasileira e sul-africana na formação da nação e da nacionalidade, simplesmente não podia existir como tal no Caribe (ou então teve ali um papel muito mais limitado, geralmente restrito às ilhas maiores $\mathrm{e}$ territórios continentais).

Em realidade, o Caribe não tem propriamente uma fronteira porque ele é todo fronteira: fronteira entre terra e mar, entre diferentes poderes coloniais, diferentes tradições, e grupos imigrantes diversos (Van Lier 1971, Knight 1990, Ribeiro 2002b). É um espaço social mediterrâneo permanentemente não-consolidado e exposto, onde as identidades estão sempre surgindo em sociedades que há muito são denominadas de "plurais" pela sociologia e história da região (Smith 1965, 1991, Dew 1978, Sankatsing 1989, Cross 1994, Knight 1995). A melhor maneira de representar as identidades caribenhas portanto não é como identidades paralelas mas separadas (como no pensamento de apartheid), nem como misturando-se num grande "nós" nacional (como nas narrativas brasileiras), mas sim como identidades que surgem de modos diferentes e complexos (Ribeiro 2002b). Não há uma totalidade maior à qual estas possam ser assimiladas (como a nação no

Universitas - Relações Int., Brasília, v. 2, n.2, p. 83-107, jul./dez. 2004 
Brasil), ou da qual possam ser segregadas (como na África do Sul). Há uma tradição de pensamento diaspórico muito forte, que Paget Henry denominou de "afro-caribenha" (Henry 2000), e que permeia toda a região. Essa tradição teria sua origem na África. Nessa tradição, a diferença cultural não surge como alteridades paralelas (como no pensamento de apartheid) ou como alteridades que se transcendem sublimando-se num todo nacional (como no pensamento brasileiro), mas como excedentes ou loci de "entrelugares", para usar dois termos emprestados a Bhabha. Isto é, as identidades aqui não estão nem compartimentadas, como na África do Sul, nem miscigenadas ou em relação entre si, como no Brasil, e muito menos relacionadas a um único campo discursivo. O Caribe é o domínio do entrelugar (inbetween) de Bhabha, a fronteira onde algo começa a surgir, algo que não estava lá antes (digamos, em identidades constituídas antes do encontro), e que não é tampouco a soma das diferentes alteridades que se juntam (Bhabha 1994, Ribeiro 2002b). Na fronteira, esse excedente dificilmente se subordina a grandes narrativas (master narratives) como as do Brasil ou da África do Sul. Como Bhabha indica, aqui a negociação da diferença cultural pode tanto estar relacionada ao conflito quanto ao consenso, e o resultado dessa negociação pode confundir noções de progresso, tradição, modernidade, desenvolvimento e nação (Blérald 1988, Múnera 1998).

Esse tipo de pensamento descentrado parece ser fundamentalmente diferente tanto do pensamento brasileiro como do sul-africano (isto a despeito das enormes divergências entre esses dois últimos). Não há um centro organizador aqui (digamos, o estadonação). Seguindo a formulação de Hayden White, poderíamos dizer que os mythòi não são as narrativas em si, mas sim as fontes para as narrativas, digamos, os princípios mitológicos que as fundamentam. Se assim for, poderíamos aventar que as narrativas brasileiras, por exemplo, inspiram-se em mythòi (White 1978). Contudo, as narrativas caribenhas, em comparação, parecem peculiarmente fragmentadas. É essa fragmentação que Benitez-Rojo denominou de um "caos de diferenças e repetições, de combinações e permutações" (Benitez-Rojo 1992:81, citado em Allen 1995: 180). Ela surpreende muito o observador externo, seja brasileiro ou sul-africano. Outro escritor

Universitas - Relações Int., Brasília, v. 2, n.2, p. 83-107, jul./dez. 2004 
caribenho - Boeli van Leeuwen, de Curaçau - capturou o fenômeno na imagem poderosa de uma geniale anarchie, ou uma admirável anarquia (Van Leeuwen 1990). Contudo, apesar de seu caráter de caleidoscópio, esse "caos" tem uma dinâmica regular ou um ar de família, emprestando agora um termo de Wittgenstein (1958). Proponho que, para capturar essa dinâmica ou ar de família, é interessante contrastar narrativas brasileiras e sul-africanas de identidade, "raça" e história com narrativas caribenhas. O método ser empregado aqui é parecido ao movimento hermenêutico proposto por Geertz (1983). Isto é, a interpretação surgiria aqui do próprio movimento heurístico entre um pensamento e outro: nenhum dos pensamentos em questão seria considerado um modelo a ser seguido. Pelo contrário, cada um servirá de contraponto para o outro. Espera-se que assim, superando fronteiras nacionais e tradições narrativas em línguas diversas e disciplinas diferentes, seja possível encontrar maneiras inusitadas e originais de interpretar a história construída das sociedades das três regiões.

\section{Referências bibliográficas}

ACHÉEN, René. 'Pour une grammaire de l'histoire anitillaise tentative de définition (Martinique-Guadeloupe, années 16351946)' in Les Temps Modernes, numéro spécial Antilles, AvrilMai 1983, pp. 1815-1835.

ADAM, Heribert and H. Giliomee. Ethnic Power Mobilized: Can South Africa Change?, Yale University Press, New Haven, 1979.

AGIER, Michel. 1995. 'Racism, culture and black identity in Brazil', Bulletin of Latin American Research 14 (3): 245-264, 1995.

ALENCASTRO, Luiz Felipe de. O Trato dos Viventes. Formação do Brasil no Atlântico Sul, São Paulo, Companhia das Letras, 2000.

ALLEN, Carolyn, 'Narrative Quilting in Banana Bottom and Voyage in the Dark', in The Reordering of Culture: Latin America, the Caribbean and Canada in the Hood. Alvina Ruprecht and Cecilia

Universitas - Relações Int., Brasília, v. 2, n.2, p. 83-107, jul./dez. 2004 
Taiana, eds., Carleton University Press, Ottawa, 1995, pp. 175186.

ALOFS, Luc and Leontine Merkies. Ken ta arubiano? Sociale integratie en natievorming op Aruba. KITLV-CARAF, Leiden, 1990.

ARAÚJO, Ricardo Benzaquen de. Guerra e Paz: Casa-Grande \& Senzala e a obra de Gilberto Freyre nos anos trinta, Editora 34, Rio, 1994.

AZEVEDO, Célia M. de Onda negra, medo branco - o negro no imaginário das elites: século XIX, Rio and São Paulo, Paz e Terra, 1987.

BAKKER, Eveline et al. Geschiedenis van Suriname: van stam tot staat. Zutphen, Walburg Pers, 2000.

BANTON, Michael. Race Relations. Basic Books, New York, 1967.

BEINART, William and Saul Dubow, eds. Segregation and Apartheid in Twentieth Century South Africa, Routledge, London and New York, 1995.

BENITEZ-ROJO, Antonio. The Repeating Island. Durham and London, Duke University Press, 1992.

BHABHA, Homi. The location of culture, Routledge, London and New York, 1994.

BIEWENGA, Ad. De Kaap de Goede Hoop: een Nederlandse vestigingskolonie 1680-1730. Prometheus/Bakker, Amsterdam, 1999.

BLÉRALD, Alain Philippe. La Question Nationale en Guadeloupe et en Martinique, L'Harmattan, Paris, 1988.

BOSMA, Ulbe and Fernando Rosa-Ribeiro. 'Java and Brazil Compared: 'Race' and Nation in Dutch and Portuguese Colonial Worlds'. Unpublished manuscript, 2002 [to appear in Sojourn].

BRAIDOTTI, Rosi. Patterns of Nomadism, Columbia University Press, New York, 1995. 
BRUIJNE, Ad de and Aart Schalkwijk, Kondreman en P'tata. Nederland als referentiekader voor Surinamers, Paramaribo, Leo Victor, 1994.

BUDDINGH', Hans. Geschiedenis van Suriname, Utrecht, Spectrum, 1995.

CHAMBERLAIN, Mary (ed). Caribbean Migration: Globalised Identities. Routledge, London and New York, 1998.

COERTZE, P.J. et al. Die oplossing van die Naturellevraagstuk in SuidAfrika: wenke ooreenkomstig die Afrikanerstandpunt van apartheid. Publicité, Johannesburg, 1943.

COETZEE, J.M. 'The Mind of Apartheid : Geoffrey Cronjé 1903 - ' in Social Dynamics 1, pp. 1-35, 1991.

COETZEE, J.M. White Writing: On the Culture of Letters in South Africa, New Haven and London, 1988.

CORRÊA, Mariza. As ilusões da liberdade - a escola Nina Rodrigues e a antropologia no Brasil, doctor's dissertation, Universidade de São Paulo, São Paulo, 1992.

CRONJÉ, Geoffrey, 'n Tuiste vir die Nageslag. Die Blywende Oplossing van Suid-Afrika se Rassevraagstukke, Johannesburg, Publicité, 1945.

CRONJÉ, Geoffrey. Afrika sonder die Asiaat, Johannesburg, 1946.

CRONJÉ, Geoffrey. Voogdyskap en apartheid, Pretoria, 1948.

CROSS, Malcolm. Ethnic Pluralism and Racial Inequality. A Comparison of Colonial and Industrial Societies, doctor's dissertation, Universiteit Utrecht, 1994

CUNHA, Euclides da. Os sertões, São Paulo, Brasiliense, 1985 (originally published in 1902).

DAMATTA, Roberto. 'A Fábula das Três Raças', in Relativizando, Petrópolis, Vozes, 1981, pp. 58-83.

Universitas - Relações Int., Brasília, v. 2, n.2, p. 83-107, jul./dez. 2004 
DAMATTA, Roberto. 'Brasil \& EUA; ou, as lições do número três', in Religião e Identidade Nacional, Rubem César Fernandes et al., Graal, Rio de Janeiro, 1988, pp. 11-26.

DAMATTA, Roberto. 'Notas sobre o racismo à brasileira', in Multiculturalismo e Racismo: Uma Comparação Brasil - Estados Unidos, Jessé Souza (ed), Paralelo 15, Brasília, 1997, pp. 69-76.

Datafolha, Racismo Cordial, São Paulo, Editora Datafolha, 1995.

DAVENPORT, T.H.R., South Africa: A Modern History, Macmillan, London, 1987.

DEBRET, Jean Baptiste and Luiz Felipe de Alencastro. Rio de Janeiro Cidade Mestiça, Companhia das Letras, 2001.

DEGLER, Carl Neither Black Nor White: slavery and race relations in Brazil and the United States, New York, Macmillan, 1971.

DE KOM, Anton. Wij slaven van Suriname, Het Wereldvenster, Bussum, The Netherlands, 1981 (originally published in 1934).

DEW, Edward. The Difficult Flowering of Suriname. Ethnicity and Politics in a Plural Society, The Hague, Nijhoff, 1978.

DU TOIT, André, and Hermann Giliomee, Afrikaner Political Thought. Analysis and Documents, Vol. 1, David Philips, Cape Town, 1983.

ELPHICK, Richard, and H. Giliomee. The Shaping of South African Society, 1652-1840, Middleton, Connecticut, Wesleyan University Press, 1988.

FANON, Frantz. Black Skins, White Masks. Grove Press, New York, 1967.

FEBRUARY, Vernon. Mind your Colour. The 'coloured' stereotype in South African literature, Londres and Boston, 1981.

FERNANDES, Rubem César et al. Brasil \& EUA: Religião $e$ Identidade Nacional, Graal, Rio de Janeiro, 1988.

FIGUEIREDO, Ângela. 1998. Novas Elites de Cor. MA Thesis. Dept. of Sociology, Universidade Federal da Bahia.

Universitas - Relações Int., Brasília, v. 2, n.2, p. 83-107, jul./dez. 2004 
FOUCAULT, Michel. Les mots et les choses; une archéologie des sciences humaines. Gallimard, Paris, 1966.

FOUCAULT, Michel. L’Archéologie du savoir. Gallimard, Paris, 1969.

FREYRE, Gilberto. Casa-Grande \& Senzala, Rio de Janeiro, José Olympio, 1984 [originally published in 1933].

FREYRE, Gilberto. Integração Portuguesa nos Trópicos/ Portuguese Integration in the Tropics: notes concerning a possible Lusotropicology, Lisbon, 1958.

GEERTZ, Clifford. 'Local Knowledge: Fact and Law in Comparative Perspective' in Local Knowledge: Further Essays in Interpretive Anthropology, Basic Books, New York, 1983.

GILIOMEE, Hermann. 'The Beginnings of Afrikaner Ethnic Consciousness, 1850-1914' in: Leroy Vail, ed., The Creation of Tribalism in Southern Africa, London and Berkeley, 1989.

GLISSANT, Edouard. Le discours antillais, Gallimard, Paris, 1981.

GOBARDHAN-RAMBOCUS, L. and Maurits S. Hassankhan, Immigratie en Ontwikkeling: emancipatie van contractanten, Anton de Kom Universiteit, Paramaribo, 1993.

GOLDIN, Ian. Making Race: the Politics and Economics of Coloured Identity in South Africa, Maskew Miller Longman, Cape Town, and Thorold's Africana Books, Johannesburg, 1987.

GOLDIN, Liliana R. (ed). Identities on the Move. Transnational Processes in North America and the Caribbean, Studies on Culture and Society, Volume 7, Institute for Mesoamerican Studies, University at Albany, Albany, New York, 1999.

GROENEBOER, Kees. Koloniale Taalpolitiek in Oost en West, Amsterdam, Amsterdam University Press, 1997.

HARRIS, Wilson. Tradition, the Writer and Society, New Beacon Books, London, 1967. 
HASENBALG, Carlos Discriminação e desigualdades raciais no Brasil, Rio de Janeiro, Graal, 1979.

HÖEFTE, Rosemarijn. Plantation Labour After the Abolition of Slavery, Ph.D. dissertation, University of Florida, Gainesville, 1987.

HÖEFTE, Rosemarijn. De betovering verbroken. De migratie van Javanen naar Suriname en het Rapport-Van Vleuten (1909), KITLV, Leiden, 1990.

HOETINK, Harry. Caribbean Race Relations: A Study of Two Variants, London, Oxford University Press, 1967.

HOETINK, Harry. Het Patroon van de oude Curaçaose samenleving, Amsterdam, Emmering, 1987.

HOFMEYER, Isabel. 'Building a nation from words: Afrikaans language, literature and ethnic identity, 1902-1924' in: Shula Marks and Stanley Trapido, ed., The Politics of Race, Class \& Nationalism in Twentieth Century South Africa, London and New York, 1987.

HUIGEN, Sigfried. De weg naar Monomotapa. Nederlandstalige representaties van geografische, historische en sociale werkelijkheden in Zuid-Afrika, Amsterdam University Press, Amsterdam, 1996.

JAMES, C.L.R. The Black Jacobins. Toussaint L'Ouverture and the San Domingo Revolution, Vintage Books, New York, 1989.

JAMES, Wilmot et al. Now That We Are Free: Coloured Communities in a Democratic South Africa. Institute for Democracy in South Africa, Cape Town, 1996.

JAMES, Winston. Holding Aloft the Banner of Ethiopia. Caribbean Radicalism in Early Twentieth Century America, Verso, London and New York, 1998.

KANNEMEYER, J.C. Geskiedenis van die Afrikaanse literatuur, Deel 1, Pretoria, 1978. 
KNIGHT, Franklin W. The Caribbean: The Genesis of a Fragmented Nationalism. New York, Oxford University Press, 1999,

KNIGHT, Franklin W. Race, Ethnicity, and Class: Forging the Plural Society in Latin America and the Caribbean, The Seventeenth Charles Edmondson Historical Lectures, Baylor University, Waco, Texas, March 27 and 28, 1995.

LEGASSICK, Martin. 'The frontier tradition in South African historiography' in: Shula Marks and Anthony Atmore eds, Economy and Society in Pre-Industrial South Africa (London 1980, pp. 44-79.

MACCRONE, I.D. Race Attitudes in South Africa. London, 1937.

MACMILLAN, W.H. Cape Colour Question. A.A. Balkema, Cape Town, 1968.

MARC, René. Por Onde o Povo Anda, doctor's dissertation, Department of History, Universidade de Brasília. Brasília, 1998.

MARKS, Shula and Stanley Trapido, ed., The Politics of Race, Class \& Nationalism in Twentieth Century South Africa, London and New York, 1987.

MATTOS, Hebe M. Das Cores do Silêncio: Os Significados da Liberdade no Sudeste Escravista - Brasil, séc. XIX, Nova Fronteira, Rio, 1995.

MELLO, Evaldo Cabral de. Rubro Veio: O Imaginário da Restauração Pernambucana, Topbooks, Rio, 1997.

MOOG, Vianna. Bandeirantes e pioneiros, Civilização Brasileira, Rio de Janeiro, 1978.

MULLER, C.F.J. Die Oorsprong van die Groot Trek, Cape Town, 1974.

MUNANGA, Kabengele. Rediscutindo a mestiçagem no Brasil, Vozes, Petrópolis, 1999. 
MÚNERA, Alfonso. El Fracaso de la Nación. Región, clase y raza en el Caribe colombiano (1717-1810), Banco de la República/El Áncora, Bogotá, 1998.

OLIVEIRA, Lúcia Lippi. A Questão Nacional na Primeira República, Brasiliense, São Paulo, 1990.

OLIVEIRA, Dijaci David et al. (ed). Relações Raciais e Grupos Socialmente Segregados, Movimento Nacional de Direitos Humanos, Goiânia, 1999.

O'MEARA, Dan. Volkskapitalisme: Class, Capital and Ideology in the Development of Afrikaner Nationalism, 1934-1948, Johannesburg, 1983.

OOSTINDIE, Gert. Het paradijs overzee. De 'Nederlandse' Carä̈ben en Nederland, Bert Bakker, Amsterdam, 1997.

ORTIZ, Renato. Cultura Brasileira \& Identidade Nacional, Brasiliense, São Paulo, 1986.

PATTERSON, Sheila. Colour and Culture in South Africa: a study of the status of the Cape Coloured People within the Union of South Africa, Routledge and Kegan Paul, Cape Town, 1953.

PEIRANO, Mariza. The anthropology of anthropology: the case of Brazil, Ph.D. dissertation, Harvard University, Cambridge, 1981.

PUNTONI, Pedro. A guerra dos bárbaros - povos indígenas e a colonização do sertão do nordeste do Brasil 1650-1720. Department of History, Universidade de São Paulo, 1998.

RENO, Fred. L'exportation de modèles d'administration opposés: le cas de la Barbade et de la Martinique, doctor's dissertation, Université Paris I (Sorbonne), 1987.

RIBEIRO, Darcy. 1995. O Povo Brasileiro. São Paulo, Companhia das Letras.

RHOODIE, N.J. 'G. Cronje se beskouing van die Suid-Afrikaanse Blank-Bantoe-problematiek, soos weerspieël in sy onmiddellik na- 
oor-logse geskrifte' in: J.E. Pieterse et al., Mens en gemeenskap, Pretoria and Cape Town, 1969.

RÖMER, R.A. Een volk op weg/Un pueblo na kaminda, Walburg, Zutphen, 1979.

ROSA, Guimarães. Grande Sertão: Veredas, José Olympio, Rio 1956.

ROSA-RIBEIRO, Fernando et al. 'Aventuras de uma branca ocidental'. Unpublished manuscript.

ROSA-RIBEIRO, Fernando. 'Apartheid" e democracia racial: raça e nação no Brasil e África do Sul', in Estudos Afro-Asiáticos, 24, 1993, pp. 95-120.

ROSA-RIBEIRO, Fernando. 'Apartheid and "Democracia Racial": South Africa and Brazil Compared'. Doctor's dissertation, Universiteit Utrecht, Utrecht, 1996a.

ROSA-RIBEIRO, Fernando. 'Diversity and Assimilation in Brazil', in Now That We Are Free: Coloured Communities in a Democratic South Africa. Wilmot James et al., Institute for Democracy in South Africa, Cape Town, 1996b.

ROSA-RIBEIRO, Fernando. 'The Dutch Diaspora: Apartheid, Boers, and Passion'. Itinerario 1, 1998, 87-106.

ROSA-RIBEIRO, Fernando. 'Un discours de fragmentation: du verzuiling à l'apartheid, Pays-Bas et Afrique du Sud, aller et retour'. Pouvoirs dans la Caraïbe, 11, 1999a, 229-251.

ROSA-RIBEIRO, Fernando. 'História e Narrativas de Etnicidade e Construção da Nação nos Países Baixos e sua Diáspora'. Boletim do Museu Paraense Emílio Goeldi, Antropologia, 15(1), 1999b, 125-161.

ROSA-RIBEIRO, Fernando. 'Racism, Mimesis and Anthropology in Brazil'. Critique of Anthropology, 20 (3), 2000, 221-241

ROSA-RIBEIRO, Fernando. 'The 'Plural Society' Revisited: Colonial and Post-Colonial Identities in Suriname, Brazil and Java', Sugarlandia, First International Workshop on Re-Thinking the 
Sugar Colony in the Asia-Pacific Region in a Global Context, International Institute of Social History, Amsterdam, 5 a 6 de julio de 2001a.

ROSA-RIBEIRO, Fernando. 'Sino-Indonesians: The Legacy of Dutch Colonialism and Colonial Nation-Building in Indonesia'. Research Project, Departamento de História, Universidade de São Paulo, 2001b.

ROSA-RIBEIRO, Fernando. 'Colonialism and the Nation-State Decentred: a View of the Non-Hispanic Caribbean from Brazil'. In: Mamadou Diouf and Ulbe Bosam (eds.) Comprendre le Pluralisme, Parcours Caraïbéens, Paris, Karthala, 2002a.

ROSA-RIBEIRO, Fernando. 'Surinam: Estado-Nación y Colonialismo en las Guyanas'. In: Región y frontera en la historia de America Latina y el Caribe. Negocios, movimientos sociales y prácticas culturales. Maria Teresa Zavala, Olga Cabrera y Jose Alfredo Uribe Salas (eds.), Universidad Michoacana de San Nicolás de Hidalgo and Universidade Federal de Goiás, San Nicolás de Hidalgo, 2002b.

ROSS, Robert. Beyond the Pale. Essays on the history of colonial South Africa, Hanover/London, 1993.

SANKATSING, Glenn. Caribbean Social Science: An Assessment, UNESCO, Caracas, 1989.

SAUNDERS, Cliff. The Mmaking of the South African Past. Major historians on race and class, Cape Town and Johannesburg, 1988.

SCHALKWIJK, Marten. Colonial State-Formation in Caribbean Plantation Societies. Structural Analysis and Changing Elite Networks in Suriname, 1650-1920, Ph.D. dissertation, Cornell University, 1994.

SHARP, John. 'Ethnic group and nation: the apartheid vision in South Africa' in: Emile Boonzaier and John Sharp ed, South African Keywords, Cape Town, 1988.

Universitas - Relações Int., Brasília, v. 2, n.2, p. 83-107, jul./dez. 2004 
SHERIFF, Robin. Negro is a Nickname that Whites Give to Blacks. Ph.D. dissertation, Department of Anthropology, City University of New York, 1997.

SILVA, Ana Rosa Clochet da. Construção da Nação e Escravidão no Pensamento de José Bonifácio 1783-1823, Editora da Universidade Estadual de Campinas, Campinas, 1999.

SKIDMORE, Thomas. Black into White: Race and Nationality in Brazilian Thought. Oxford University Press, 1974.

SKIDMORE, Thomas. 'Bi-racial USA vs. multi-racial Brazil: is the contrast still valid?', Journal of Latin American Studies, 25, 2: 373-86, 1993.

SMEULDERS, T.F. Papiamentu en onderwijs: veranderingen in beeld en betekenis van de volkstaal op Curaçao, doctor's dissertation, Universiteit Utrecht, 1987.

SMITH, M.G. The Plural Society in the British West Indies, University of California Press, Berkeley, 1968.

SMITH, M.G. Pluralism, Politics and Idelogy in the Creole Caribbean, Vera Rubin Caribbean Series Number One, Research Institute for the Study of Man, New York, 1991.

SOUZA, Jessé (ed). Multiculturalismo e Racismo: Uma Comparação Brasil - Estados Unidos, Paralelo 15, Brasília, 1997.

SOUZA, Candice Vidal de. A Pátria Geográfica, Editora Universidade Federal de Goiás, Goiânia, 1998.

SUÁREZ, Mireya. 1998. "Sertanejo: Um Personagem Mítico". Sociedade e Cultura, 1 (1): 29-39, jan./jun. 1998.

TAUSSIG, Michael. Mimesis and Alterity: A Particular History of the Senses, Routledge, New York and London, 1993.

TAUSSIG, Michael. The Magic of the State, Routledge, New York and London, 1997.

THOMPSON, Leonard. The Political Mythology of Apartheid, Yale University Press, New Haven, 1985.

Universitas - Relações Int., Brasília, v. 2, n.2, p. 83-107, jul./dez. 2004 
TORRES, Alberto. O Problema Nacional Brasileiro, Editora Universidade de Brasília, Brasília, 1982 [originally published in 1914].

VAN ALLER, H.B. Van kolonie tot rijksdeel: de staatkundige geschiedenis van de Nederlandse Antillen en Aruba, WoltersNoordhoff, Groningen, 1994.

VAN DEN BERGHE, Pierre. South Africa: a study in conflict, Middletown, Conn., Wesleyan University Press, 1965.

VAN DER MERWE, P.J. Die noordwaartse beweging van die Boere voor die Groot Trek (1770-1842), The Hague, W.P. van Stockum, 1937

VAN JAARSVELD, F.A. Die Afrikaner en sy Geskiedenis, Cape Town, 1959 (also available in English: The Afrikaner Interpretation of South African History, Cape Town, 1961).

VAN JAARSVELD, F.A. 'Oor vertolking en hervertolking in ons geskiedskrywing' in Geskiedkundige Verkenninge, Van Schaik, Pretoria, 1974.

VAN LEEUWEN, Boeli. Geniale anarchie. In de Knipscheer, Amsterdam, 1990.

VAN STIPRIAAN, Alex. Surinaams Contrast. Roofbouw en overleven in een Caraïbische plantagekolonie 1750-1863, Leiden, KITLV, 1993.

VELHO, Otávio. Capitalismo Autoritário e Campesinato. Difel, São Paulo, 1976.

VENTURA, Roberto. Estilo Tropical: História Cultural e Polêmicas Literárias no Brasil, Companhia das Letras, São Paulo, 1991.

VOGT, Carlos and Peter Fry. Cafundó. A África no Brasil, Companhia das Letras, São Paulo, 1996.

WALKER, Eric, 'The Frontier Tradition in South Africa,' lecture delivered at Rhodes House, Oxford, 5th March 1930.

Universitas - Relações Int., Brasília, v. 2, n.2, p. 83-107, jul./dez. 2004 
WEBER, João Hernesto. A Nação e o Paraíso. A Construção da Nacionalidade na Historiografia Literária Brasileira, Editora da UFSC, Florianópolis, 1997.

WHITE, Hayden. Tropics of Discourse: Essays in Cultural Criticism. Johns Hopkins University Press, Baltimore and London, 1978.

WILSON-TAGOE, Nana. Historical Thought and Literary Representation in West Indian Literature, University Press of Florida, The Press University of the West Indies, James Currey, London, 1998.

WITTGENSTEIN, Ludwig. Philosophical Investigations, Prentice Hall, Englewood Cliffs, New Jersey, 1958.

WORDEN, Nigel. Slavery in Dutch South Africa, Cambridge University Press, Cambridge, 1985).

WORDEN, Nigel. The Making of South African Society: Conquest, Segregation and Apartheid, Blackwell, Londres, 1994.

YOUNG-BRUEHL, Elizabeth. The Anatomy of Prejudices. Cambridge University Press, Cambridge, Massachussetts.

Universitas - Relações Int., Brasília, v. 2, n.2, p. 83-107, jul./dez. 2004 